\title{
Enhancing fits of SMEFT Wilson coefficients in the top-quark sector
}

\section{Stefan Bißmann, ${ }^{a}$ Johannes Erdmann, ${ }^{a}$ Cornelius Grunwald, ${ }^{a, *}$ Gudrun Hiller ${ }^{a}$ and Kevin Kröninger ${ }^{a}$}

${ }^{a}$ TU Dortmund University,

Otto-Hahn-Str. 4, 44227 Dortmund, Germany

E-mail: stefan.bissmann@tu-dortmund.de, johannes.erdmann@tu-dortmund.de, cornelius.grunwald@tu-dortmund.de, ghiller@physik.uni-dortmund.de, kevin.kroeninger@tu-dortmund.de

Over the last years, various efforts were made for interpreting measurements of top-quark observables in the context of the Standard Model Effective Field Theory (SMEFT). We point out two aspects for enhancing fits constraining Wilson coefficients of dimension-six operators that should be considered when aiming towards a global fit of SMEFT coefficients in the top-quark sector. On the one hand, we discuss the advantages of combining measurements from top-quark and $B$ physics for constraining top-quark couplings. Considering $\sigma(t \bar{t} \gamma)$ together with $\operatorname{BR}\left(\bar{B} \rightarrow X_{s} \gamma\right)$ as an example, we present the steps necessary for including observables from different energy scales in a combined fit and highlight the benefits of this approach. On the other hand, we discuss the importance of taking into account correlations between the uncertainties of measurements by demonstrating the impact correlations can have on the constraints of Wilson coefficients.

40th International Conference on High Energy physics - ICHEP2020

July 28 - August 6, 2020

Prague, Czech Republic (virtual meeting)

${ }^{*}$ Speaker 


\section{BSM searches with effective field theories}

In the search for physics beyond the Standard Model (BSM), no direct detection of BSM phenomena could be reported until today and approaches to indirectly examine higher energy regimes using effective field theories (EFT) are becoming very popular in top-quark physics, complementing direct searches at the $\mathrm{TeV}$ scale. The Standard Model Effective Field Theory (SMEFT) is an extension of the SM Lagrangian that parametrizes the low-energy effects of BSM phenomena in a model-independent way. This is achieved by introducing higher-dimensional operators $O_{i}$ with corresponding Wilson coefficient $C_{i}$, suppressed by orders of the energy scale of BSM physics $\Lambda$. Obeying lepton and baryon number conservation, the leading BSM contributions arise from dimension-six operators:

$$
\mathcal{L}_{\mathrm{SMEFT}}=\mathcal{L}_{\mathrm{SM}}+\sum_{i} \frac{1}{\Lambda^{2}} C_{i}^{(6)} O_{i}^{(6)}+O\left(\Lambda^{-4}\right)
$$

Deviations of the Wilson coefficients from zero would be a signal of BSM physics and constraining the Wilson coefficients therefore allows model-independent BSM searches.

In the following, we present two studies investigating aspects for enhancing SMEFT fits of Wilson coefficients in the top-quark sector.

\section{Combination of observables from top-quark and flavor physics}

As dimension-six operators affecting top-quark physics can also contribute to $B$ physics processes, e.g. via top-quark loops, we investigate the possibility of enhancing fits of the top-quark sector of SMEFT by combining observables from top-quark and $B$ physics [1]. Specifically, we constrain couplings of the top quark to the gauge bosons using measurements of $t \bar{t} \gamma$ production cross sections and of the $\bar{B} \rightarrow X_{s} \gamma$ branching fraction. We consider only dimension-six operators involving third generation quarks and bosonic fields:

$$
O_{u B}=\left(\bar{q}_{L} \sigma^{\mu \nu} u_{R}\right) \tilde{\varphi} B_{\mu \nu}, \quad O_{u G}=\left(\bar{q}_{L} \sigma^{\mu \nu} T^{A} u_{R}\right) \tilde{\varphi} G_{\mu \nu}^{A}, \quad O_{u W}=\left(\bar{q}_{L} \sigma^{\mu \nu} \tau^{I} u_{R}\right) \tilde{\varphi} W_{\mu \nu}^{I},
$$

where $q_{L}$ is the $S U(2)$ doublet and $u_{R}$ is the up-type singlet. Corresponding operators with third generation down-quarks are omitted as they are suppressed by a factor $m_{b} / m_{t}$. The Higgs-doublet is denoted as $\varphi$ and $B_{\mu \nu}, W_{\mu \nu}^{I}$ and $G_{\mu \nu}^{A}$ are the gauge field strength tensors. We use single-lepton and dilepton channel measurements of fiducial $t \bar{t} \gamma$ cross sections performed by ATLAS at $13 \mathrm{TeV}$ [2] and a combination of $\operatorname{BR}\left(\bar{B} \rightarrow X_{s} \gamma\right)$ measurements performed by the Heavy Flavor Averaging Group (HFLAV) [3].

To determine the dependence of the fiducial $t \bar{t} \gamma$ cross sections on the dimension-six operators, we employ MadGraph5_aMC@NLO [4] together with the dim6top_LO UFO model [5]. We compute the cross sections at several sampling points in the space of Wilson coefficients and perform an event selection according to the fiducial phase space definition at each of them. Using a quadratic interpolation of the total cross section and of the fiducial acceptance, we determine the single-lepton and dilepton fiducial cross sections as a function of the three Wilson coefficients $C_{u B}, C_{u G}, C_{u W}$.

To determine the dependence of $\operatorname{BR}\left(\bar{B} \rightarrow X_{s} \gamma\right)$ on the SMEFT Wilson coefficients, we utilize that BSM contributions to $b \rightarrow s \gamma$ transitions can be described in terms of the Weak Effective 
Theory (WET), which is defined at energy scales below the mass of the $W$ boson. To use WET calculations for this, we need to match the SMEFT Lagrangian to the WET Lagrangian at a matching scale $\mu_{w}$. Therefore, we apply renormalization group equations (RGEs) to evolve SMEFT from the energy scale of top-quark measurements $\mu_{t} \sim m_{t}$ to the matching scale $\mu_{w} \sim m_{W}$, where we then match the SMEFT coefficients onto the WET coefficients using matching conditions calculated at one-loop level [6]. Using WET RGEs, we evolve the coefficients from $\mu_{W}$ to $\mu_{b}$, yielding the dependence between the SMEFT Wilson coefficients at scale $\mu_{t}$ and the WET Wilson coeffiecients at scale $\mu_{b}$. With this matching of SMEFT to WET, we can formulate the dependence of $\operatorname{BR}\left(\bar{B} \rightarrow X_{s} \gamma\right)$ on the Wilson coefficients of the operators in Eq. (2), as the calculation of BSM contributions to $\operatorname{BR}\left(\bar{B} \rightarrow X_{s} \gamma\right)$ is known in terms of WET Wilson coefficients [7].

Following a Bayesian approach using a new implementation of the EFTfitter [8] based on BAT.j1 [9], we constrain the Wilson coefficients. We perform three different fits, using only the cross-section measurements, using only the branching ratio measurement and using the combination of both. The resulting constraints on the rescaled Wilson coefficients $\tilde{C}_{i}=\frac{v^{2}}{\Lambda^{2}} C_{i}$, where $v=246 \mathrm{GeV}$, are shown in Fig. 1 for the marginalized 2D posterior probability distribution of $\tilde{C}_{u B}$ vs. $\tilde{C}_{u G}$ and $\tilde{C}_{u B}$ vs. $\tilde{C}_{u W}$. In both plots, the smallest intervals containing $90 \%$ posterior probability are shown for the fits using only one of the observables and for the combined fit using the top-quark and $B$ observables. In both plots of Fig. 1 it is visible that when using only one of the observables, the
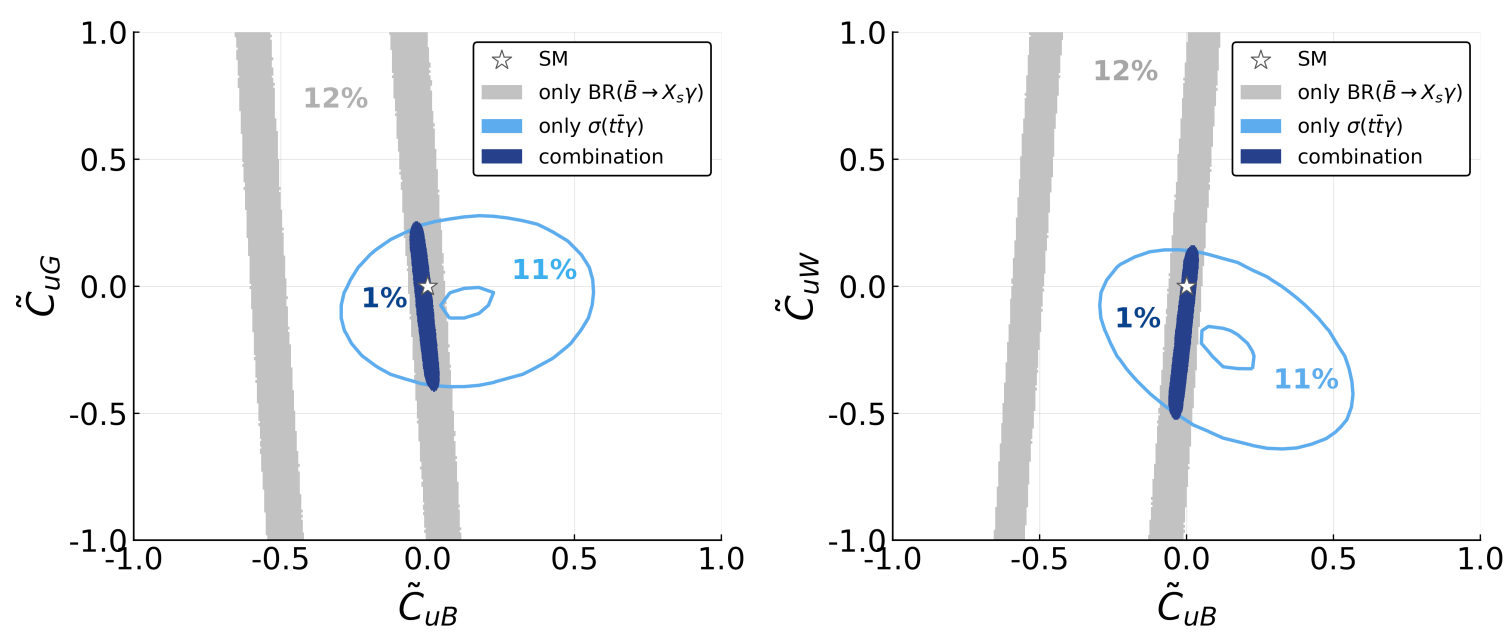

Figure 1: Constraints on the Wilson coefficients from fits using only $\operatorname{BR}\left(\bar{B} \rightarrow X_{s} \gamma\right)$ observables, only $\sigma(t \bar{t} \gamma)$ and from the combined fit, adapted from [1]. Shown are the marginalized 2D areas containing $90 \%$ posterior probability.

resulting constraints have a size of more than $10 \%$ of the allowed region $\tilde{C}_{i} \in[-1,1]$, while when combining both, the size of this region decreases to only $1 \%$. Due to the different sensitivities of the top-quark and $B$ physics observables to the dimension-six operators, their combination allows to resolve ambiguous solutions and leads to improved constraints by up to an order of magnitude in this example. 


\section{Impact of correlated uncertainties on the constraints of Wilson coefficients}

When performing global fits of SMEFT Wilson coefficients, various measurements of multiple observables coming from different experiments are included. Uncertainties of these measurements, however, can be correlated. Unfortunately, estimating correlations between the uncertainties of all measurements included is not straightforward and therefore such correlations are often neglected in global fits. We study the influence of correlated uncertainties on the constraints of Wilson coefficients obtained in SMEFT fits using measurements of different observables from single topquark production and top-quark decay [10]. We compare two scenarios: A fit using only the published correlations and a fit where the available correlations are included together with a set of simplifying assumptions for further (undetermined) correlations. In our studies we consider $t$-channel single top-quark production cross sections, $W$ boson helicity fractions and the top-quark decay width. At $O\left(\Lambda^{-2}\right)$ the operators contributing to the processes considered are

$$
\begin{array}{ll}
O_{\varphi q}^{(3)}=i\left(\varphi^{\dagger} \overleftrightarrow{D}_{\mu}^{I} \varphi\right)\left(\bar{q}_{L} \gamma^{\mu} \tau^{I} q_{L}\right), & O_{t W}=\left(\bar{q}_{L} \sigma^{\mu \nu} \tau^{I} t_{R}\right) \tilde{\varphi} W_{\mu \nu}^{I}, \\
O_{q q}^{(1)}=\left(\bar{q}_{L} \gamma_{\mu} q_{L}\right)\left(\bar{q}_{L} \gamma^{\mu} q_{L}\right), & O_{q q}^{(3)}=\left(\bar{q}_{L} \gamma_{\mu} \tau^{I} q_{L}\right)\left(\bar{q}_{L} \gamma^{\mu} \tau^{I} q_{L}\right) .
\end{array}
$$

Neglecting contributions proportional to masses much smaller than the top-quark mass, the observables depend on three coefficients:

$$
\tilde{C}_{\varphi q}^{(3)}, \tilde{C}_{t W} \text { and } \tilde{C}_{q q}=\tilde{C}_{q q}^{(3) 1133}+\frac{1}{6}\left(\tilde{C}_{q q}^{(1) 1331}-\tilde{C}_{q q}^{(3) 1331}\right) .
$$

We constrain these three Wilson coefficients using a total of 55 measurements of 41 different observables, counting each bin of a differential distribution as an individual observable. The measurements include total and differential single (anti-)top quark $t$-channel production cross sections measured by ATLAS and CMS at 7, 8 and $13 \mathrm{TeV}$, measurements of $W$ boson helicity fractions from CDF, D0, ATLAS and CMS at 1.96, 7, 8 and $13 \mathrm{TeV}$ as well as the top-quark decay width as measured by ATLAS at $8 \mathrm{TeV}$ (see Tab. 1 in Ref. [10]). We summarize the uncertainties of all measurements into three categories: statistical, systematic and theory uncertainties. We compute the $t$-channel cross sections using MadGraph5_aMC@NLO with the dim6top_LO UFO model and perform interpolations using both a linear and a quadratic ansatz,

$$
\sigma=\sigma_{\mathrm{SM}}+\sum_{i} \tilde{C}_{i} \sigma_{i} o \quad \text { and } \quad \sigma=\sigma_{\mathrm{SM}}+\sum_{i} \tilde{C}_{i} \sigma_{i}+\sum_{i \leq j} \tilde{C}_{i} \tilde{C}_{j} \sigma_{i j}
$$

to parametrize the dependencies of the cross sections on the Wilson coefficients. BSM contributions to top-quark decay observables are determined analogously to Ref. [11], also considering quadratic contributions.

Constraints on the Wilson coefficients are again derived using EFTfitter. For our so-called no correlation scenario, the measurements and their uncertainties are included in the fit together with the available correlations, comprising mainly statistical correlations between the bins of differential distributions and between the $W$ boson helicity fractions. The resulting constraints on the Wilson coefficients are given in the left plot of Fig. 2, where the marginalized smallest intervals containing $95 \%$ of the posterior probability are shown together with the corresponding best fit values. The 
constraints obtained using the linear and the quadratic ansatz show very good agreement for all three coefficients and no significant deviations from the SM are observed.

As for the correlations of systematic and theory uncertainties no information is available, we use a set of simplifying assumptions to parametrize presumable correlations between these uncertainties, forming our best guess scenario. We parametrize the systematic correlation matrix using a correlation coefficient $\rho_{\text {sys }}$ and assume uncertainties of measurements performed at the same experiment and at the same energy to be correlated with this coefficient. Measurements performed at different energies we assume to be less strongly correlated, setting the corresponding correlation coefficients to $\frac{\rho_{\mathrm{sys}}}{2}$. Uncertainties of measurements from different experiments are assumed to be uncorrelated. A similar set of assumptions is applied for the correlation matrix of the theory uncertainties, using a parametrization with the correlation coefficients $\rho_{\text {th }}$ and $\frac{\rho_{\text {th }}}{2}$. Our no correlation scenario therefore corresponds to setting $\rho_{\text {sys }}=\rho_{\text {th }}=0$, while for the best guess correlation scenario we choose $\rho_{\text {sys }}=\rho_{\text {th }}=0.9$. The resulting constraints from the fit in the best guess scenario are shown in the right plot of Fig. 2. It is again visible that the quadratic and the linear ansatz yield very similar results. It can be noticed that in the best guess scenario deviations from the SM increase for all three Wilson coefficients, especially for $\tilde{C}_{\varphi q}^{(3)}$, having a deviation of about $4.7 \sigma$. As the correlations lead to a deformation of the three-dimensional posterior distribution, the width of the marginalized $95 \%$ intervals in Fig. 2 increases for $\tilde{C}_{\varphi q}^{(3)}$ while it decreases for $\tilde{C}_{q q}$.

When varying the values of $\rho_{\text {sys }}$ and $\rho_{\text {th }}$ from 0.0 to 0.9 , we find a continuous and consistent behavior of the constraints as a function of the correlation coefficients. When scaling the statistical uncertainties to an integrated luminosity of $300 \mathrm{fb}^{-1}$, we find that the deviations from the SM in the case of the best guess scenario increase even more up to about $12 \sigma$.
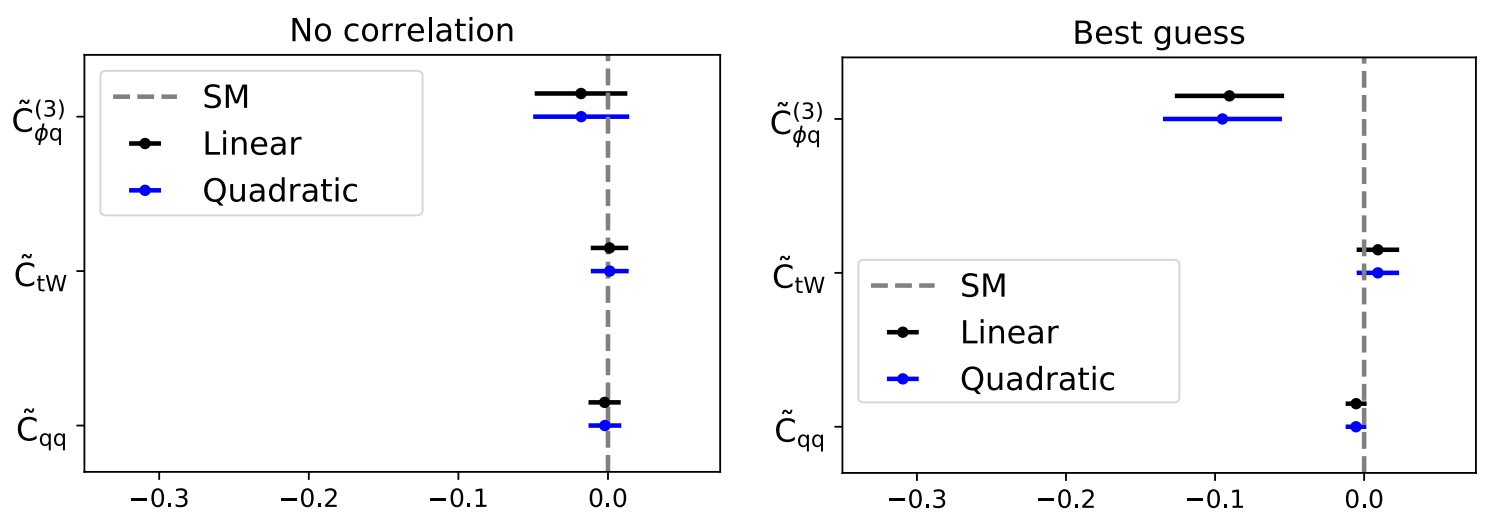

Figure 2: Marginalized smallest intervals containing $95 \%$ posterior probability and best fit values for the no correlation (left) and best guess correlation (right) scenarios, adapted from [10].

\section{Conclusions}

With the studies performed, we investigated two elements to consider for enhancing fits of SMEFT Wilson coefficients affecting the top-quark sector. On the one hand, we demonstrated that combining measurements from flavor physics with measurements from the top-quark physics can significantly improve the constraints on the SMEFT Wilson coefficients and resolve ambiguities 
due to the different sensitivities of the observables. On the other hand, we investigated the influence of correlated uncertainties on the constraints of Wilson coefficients. We revealed that correlated sources of uncertainties can have a significant impact on the constraints and that their influence becomes even stronger when future measurements with smaller statistical uncertainties are considered. Including correlations between the uncertainties might then decide whether deviations from the SM predictions are observed or not. The results of our studies show that both aspects examined should be taken into account in global fits of SMEFT Wilson coefficients.

\section{References}

[1] S. Bißmann, J. Erdmann, C. Grunwald, G. Hiller, and K. Kröninger, Constraining top-quark couplings combining top-quark and B decay observables, The European Physical Journal C 80 (2020).

[2] M. Aaboud et al., Measurements of inclusive and differential fiducial cross-sections of $t \bar{t} \gamma$ production in leptonic final states at $\sqrt{s}=13 \mathrm{TeV}$ in ATLAS,

The European Physical Journal C 79 (2019).

[3] HFLAV Collaboration, 2019, uRL: https://hflav-eos . web . cern.ch/hflaveos/rare/April2019/RADLL/OUTPUT/HTML/radll_table3.html.

[4] J. Alwall et al., The automated computation of tree-level and next-to-leading order differential cross sections, and their matching to parton shower simulations, Journal of High Energy Physics 2014 (2014).

[5] J. A. A. Saavedra et al., Interpreting top-quark LHC measurements in the standard-model effective field theory, 2018, arXiv: 1802.07237 [hep-ph].

[6] J. Aebischer, A. Crivellin, M. Fael, and C. Greub, Matching of gauge invariant dimension-six operators for $b \rightarrow s$ and $b \rightarrow c$ transitions, Journal of High Energy Physics 2016 (2016).

[7] M. Misiak et al., Updated Next-to-Next-to-Leading-Order QCD Predictions for the Weak Radiative B-Meson Decays, Physical Review Letters 114 (2015).

[8] N. Castro, J. Erdmann, C. Grunwald, K. Kröninger, and N.-A. Rosien, EFTfitter: a tool for interpreting measurements in the context of effective field theories, The European Physical Journal C 76 (2016).

[9] O. Schulz et al., BAT.jl - A Julia-based tool for Bayesian inference, 2020, arXiv: 2008.03132 [stat.CO].

[10] S. Bißmann, J. Erdmann, C. Grunwald, G. Hiller, and K. Kröninger, Correlating uncertainties in global analyses within SMEFT matters, 2019, arXiv: 1912.06090 [hep-ph].

[11] C. Zhang and S. Willenbrock, Effective-field-theory approach to top-quark production and decay, Physical Review D 83 (2011). 